Ti me- dependent measur ement of the mode compet i t i on phenomena anong I ongi tudi nal modes i $n$ I ong- wavel engt $h$ I aser s

\begin{tabular}{|l|l|}
\hline 著者 & $\begin{array}{l}\text { Yanada M nor u, I shi mor i Wat ar u, Sakaguchi } \\
\text { H r onobu, Ahmed Noust af a }\end{array}$ \\
\hline $\begin{array}{l}\text { j our nal or } \\
\text { publ i cat i on t i t l e }\end{array}$ & I EEE J OURNAL OF QUANTUM ELECTRON CS \\
\hline vol une & 39 \\
\hline number & 12 \\
\hline page r ange & 1548 1554 \\
\hline year & $2003-12-01$ \\
\hline URL & ht t p: //hdl . handl e. net /2297/1813 \\
\hline
\end{tabular}




\title{
Time-Dependent Measurement of the Mode-Competition Phenomena Among Longitudinal Modes in Long-Wavelength Lasers
}

\author{
Minoru Yamada, Member, IEEE, Wataru Ishimori, Hironobu Sakaguchi, and Moustafa Ahmed, Member, IEEE
}

\begin{abstract}
Modal behaviors of an InGaAsP-InP Fabry-Perot laser emitting in a long-wavelength region were experimentally examined. The authors had predicted theoretically that the mode-competition phenomena induce quasi-periodic hopping among several longitudinal modes, which reveal multimode-like output spectra as the time-averaged spectra in long-wavelength lasers. In this paper, experimental measured data of the time variations of photon number and their frequency spectra in addition to the longitudinal mode spectra are reported together with theoretically simulated results. The previous theoretical predictions were well proved by these experimental measurements.
\end{abstract}

Index Terms-Experimental measurement, InGaAsP-InP lasers, mode-competition phenomena, mode hopping, multimode.

\section{INTRODUCTION}

$\mathbf{S}$ EMICONDUCTOR lasers are popularly used as optical sources in optical fiber communication systems and in the optical disk system. For wider application of optical subscriber networks such as the fiber to the home (FTTH) system, InGaAsP-InP lasers with Fabry-Perot (FP) type structure will be mostly used as emitting sources because of low cost of the device and suitable wavelength for the optical fiber system.

The single longitudinal mode operation has been achieved in AlGaAs-GaAs FP-type lasers. Theoretical background for the single-mode operation was firmly given in terms of the strong gain suppression effect for nonlasing modes when the transverse modes are well controlled under CW operation [1]-[6].

However, difficulties for getting the single longitudinal mode operation in InGaAsP-InP FP-type lasers were pointed out experimentally even when the transverse modes were well controlled [7], [8].

As a possible mechanism showing the multilongitudinal mode operation, Ogita indicated an effect due to the asymmetric gain suppression, where modes on the shorter wavelength side of the lasing mode are suppressed but those on the longer wavelength side are enhanced [9].

Manuscript received March 28, 2003; revised September 12, 2003.

M. Yamada, W. Ishimori, and H. Sakaguchi are with the Department of Electrical and Electronic Engineering, Faculty of Engineering, Kanazawa University, Kanazawa, 920-8667 Japan (e-mail: myamada@t.kanazawa-u.ac.jp).

M. Ahmed is with the Department of Physics, Faculty of Science, Minia University, 61519 El-Minia, Egypt (e-mail: m.farghal@link.net).

Digital Object Identifier 10.1109/JQE.2003.819546
This model was re-examined by Ahmed and Yamada more precisely by adding intrinsic quantum noise sources which induce instability in the lasing operation and was found that the lasing modes hop almost periodically among several longitudinal modes and show multimode-like spectrum in time-averaged output [10]. Namely, the laser operates with a single mode instantaneously, but the lasing mode hops to another mode soon on the longer wavelength side by the asymmetric gain suppression. This kind of hopping continues over several longitudinal modes. Then the lasing mode switches back to a mode on the shorter wavelength side because of reduction of the linear gain in further longer wavelength region. Such phenomena are remarkable in long-wavelength lasers made of InGaAsP-InP because the asymmetric gain distribution attains values higher than the linear gain distribution along optical frequencies (longitudinal mode distribution) [11]-[14].

In this paper, we report experimental evidences of the abovementioned phenomena in a 1.3- $\mu \mathrm{m} \mathrm{InGaAsP-InP} \mathrm{FP} \mathrm{laser.} \mathrm{Time}$ variations of output power of each longitudinal mode and total intensity by all lasing modes as well as optical frequency spectrum of the output light and intensity noise were experimentally measured. The measured results were in good agreement with theoretical simulations based on our previously published model [10].

This paper is organized as follows. In the next section, the mechanism of the predicted multimode-like oscillation is reviewed. In Section III, experimental examinations are shown comparing with the theoretical simulations. Conclusions of this work are given in Section IV.

\section{Mechanism of the Multimode-Like Oscillation}

\section{A. Theoretical Model}

Variations of the photon number $S_{p}$ of a lasing mode $p$ and the injected electron number $N$ are given mathematically as [10], [15]

$$
\begin{aligned}
\frac{d S_{p}}{d t} & =\left(G_{p}-G_{\mathrm{th}}\right) S_{p}+\frac{a \xi N}{V\left\{\left[\frac{2\left(\lambda_{p}-\lambda_{0}\right)}{\delta \lambda}\right]^{2}+1\right\}}+F_{p}(t) \\
G_{p} & =A_{p}-B S_{p}-\sum_{q \neq p}\left\{D_{p(q)}+H_{p(q)}\right\} S_{q}
\end{aligned}
$$




$$
\frac{d N}{d t}=-\sum_{p} A_{p} S_{p}-\frac{N}{\tau_{s}}+\frac{I}{e}+F_{N}(t)
$$

Here, $G_{p}$ is the modal gain where the saturation effects are taken into account. $A_{p}$ is the linear gain coefficient, $B$ is the selfsaturation coefficient, and $D_{p(q)}$ and $H_{p(q)}$ are the symmetric and the asymmetric cross-saturation coefficients from mode $q$ to $p$, respectively, given as [6], [14]

$$
\begin{aligned}
A_{p}= & \frac{a \xi}{V}\left\{N-N_{g}-b V\left(\lambda_{p}-\lambda_{0}\right)^{2}\right\} \\
B= & \frac{9}{2} \frac{\pi c}{\epsilon_{0} n_{r}^{2} \hbar \lambda_{0}}\left(\frac{\xi \tau_{i n}}{V}\right)^{2} a\left|R_{c v}\right|^{2}\left(N-N_{s}\right) \\
D_{p(q)}= & \frac{4}{3} \frac{B}{\left(\frac{2 \pi c \tau_{\text {in }}}{\lambda_{p}^{2}}\right)^{2}\left(\lambda_{p}-\lambda_{q}\right)^{2}+1} \\
H_{p(q)}= & \frac{3}{4}\left(\frac{a \xi}{V}\right)^{2}\left(N-N_{g}\right) \\
& \cdot \frac{\frac{1}{\tau_{s}}+\frac{3}{2} \frac{a \xi}{V} S+\alpha \frac{2 \pi c}{\lambda_{p}^{2}}\left(\lambda_{q}-\lambda_{p}\right)}{\left(\frac{1}{\tau_{s}}+\frac{3}{2} \frac{a \xi}{V} S\right)^{2}+\left(\frac{2 \pi c}{\lambda_{p}^{2}}\right)^{2}\left(\lambda_{q}-\lambda_{p}\right)^{2}}
\end{aligned}
$$

where $a$ is the slope (or tangential) coefficient to characterize the linear gain coefficient, $\xi$ is the field confinement factor of the optical field into the active region, $V$ is the volume of the active region, $b$ is a coefficient giving the wavelength dispersion of the linear gain, $\lambda_{0}$ is the center wavelength of the linear gain, $N_{g}$ is the transparent electron number, $\tau_{\text {in }}$ is the intraband relaxation time of the electron wave, $R_{c v}$ is the dipole moment, $N_{s}$ is an electron number characterizing the saturation coefficient $B, c$ is the speed of light in free space, $n_{r}$ is the refractive index, $\tau_{s}$ is the electron lifetime, $S\left(=\sum_{p} S_{p}\right)$ is the total photon number summed for all existing modes, and $\alpha$ is the linewidth enhancement factor [16].

The term $a \xi N / V\left\{\left[2\left(\lambda_{p}-\lambda_{0}\right) / \delta \lambda\right]^{2}+1\right\}$ of (1) accounts for inclusion of the spontaneous emission into mode $p$, where $\delta \lambda$ is the half width of the spontaneous emission profile. $I$ and $e$ in (3) are the injection current and the electron charge, respectively. $F_{p}(t)$ and $F_{N}(t)$ in (1) and (3) are generating term of the fluctuation caused by the spontaneous emission [10], [17].

The coefficient $H_{p(q)}$ of (7) is approximated as

$$
H_{p(q)} \approx \frac{3 \lambda_{p}^{2}}{8 \pi c}\left(\frac{a \xi}{V}\right)^{2} \frac{\alpha\left(N-N_{g}\right)}{\lambda_{q}-\lambda_{p}}
$$

when condition of

$$
\frac{1}{\tau_{s}}+\frac{3}{2} \frac{a \xi}{V} S \ll \frac{2 \pi c}{\lambda_{p}^{2}}\left|\lambda_{q}-\lambda_{p}\right|
$$

exists. This condition is held in almost all conventional lasers.

Since the gain saturation by $D_{p(q)}$ gives almost symmetric profile for the optical wavelength difference $\left|\lambda_{q}-\lambda_{p}\right|, D_{p(q)}$ is called the symmetric cross-saturation coefficient. On the other hand, the coefficient $H_{p(q)}$ of (7) or (8) works to suppress the lasing gain for the modes on the short-wavelength side, but works to increase the lasing gain of the modes on the longer wavelength side. Then, $H_{p(q)}$ is called the asymmetric cross-saturation coefficient [11]-[14]. The effect of $H_{p(q)}$ is pronounced when the linewidth enhancement factor $\alpha$ is large and the wavelength dispersion of the linear gain is gradual, that is, the coefficient $b$ is small in (4).

$G_{\text {th }}$ in (1) is the threshold gain level and is determined with the absorption loss coefficient $\kappa$ of the laser and the mirror loss as

$$
G_{\mathrm{th}}=\frac{c}{n_{r}}\left\{\kappa+\frac{1}{2 L} \ln \frac{1}{R_{f} R_{b}}\right\}
$$

where $R_{f}$ and $R_{b}$ are power reflectivities at the front and back facets, respectively.

The electron lifetime $\tau_{s}$ is examined by the next equation with the effective rate of the spontaneous recombination $B_{\text {eff }}$ as

$$
\frac{1}{\tau_{s}} \approx B_{\text {eff }} \frac{N}{V}
$$

We label here the mode number to be $p=0, \pm 1$, $\pm 2, \ldots, \pm M$, where the mode $p=0$ is at the center wavelength $\lambda_{0}$ of the linear gain. The lasing modes on the shorter wavelength side, $\lambda_{p}<\lambda_{0}$, are indicated with negative numbers $-M \leq p<0$, while those on the longer side, $\lambda_{p}>\lambda_{0}$, are indicated with positive numbers $0<p \leq M$. The total number of the modes counted in the simulation is $2 M+1$.

Numerical simulations of the lasing operation were performed utilizing the rate equations (1) and (3) based on the manner described in [10]. The total number of modes counted in the simulation is 15 , that is, $M=7$.

The numerical values of parameters used in the simulations are listed in Table I supposing a 1.3- $\mu \mathrm{m}$ InGaAsP-InP FP laser.

\section{B. Periodic Hopping and the Multimode-Like Oscillation}

Dynamics of the laser can be analyzed by (1)-(11). Here, we will give a qualitative explanation for periodic mode hopping caused by the asymmetric cross saturation $H_{p(q)}$ with Fig. 1(a)-(d).

When a laser is operated with higher injection current $I$ than the threshold current level $I_{\text {th }}$, the single-mode operation with mode $p=0$ should be assumed because of the highest value of the linear gain $A_{p}$. The saturated gain of this mode $p=0$ is $G_{p} \approx G_{\text {th }}$ as indicated by the solid circle in Fig. 1(a). Gains of other modes on the shorter wavelength side are suppressed to lower values than the threshold gain level $G_{\text {th }}$ as indicated with hollow squares, but those on the longer wavelength side are relatively enhanced due to a negative value of $H_{p(q)}$ especially at neighbor mode to the lasing mode. In the case of Fig. 1(a), gain of mode $p=+1$ becomes higher than $G_{\text {th }}$ as indicated with the solid square. Then, the lasing mode hops (jumps) from $p=0$ to $p=+1$, and the operating state changes to Fig. 1(b). At this instance, gain of the mode $p=+1$ keeps $G_{p} \approx G_{\text {th }}$ and the laser shows the single-mode operation with the mode $p=$ +1 . Gains of almost all other modes except the mode $p=+2$ are suppressed by the lasing mode. However, the gain of mode $p=+2$ becomes higher than $G_{\mathrm{th}}$. Then, the lasing mode hops from $p=+1$ to $p=+2$ and the gain profile becomes like that shown in Fig. 1(c).

Such hopping (or jumping) to longer wavelength side continues until the linear gain $A_{p}$ becomes sufficiently small. Fig. 1(d) is the case that gain $G_{p}$ on the longer wavelength side 
TABLE I

VALUES OF PARAMETER USED IN THE Simulations OF AN INGAAsP LASER

\begin{tabular}{|c|c|c|}
\hline Parameter & Value & Unit \\
\hline Slope of linear gain $a$ & $9.20 \times 10^{-12}$ & $m^{3} s^{-1}$ \\
\hline Electron number at transparency $N_{g}$ & $8.41 \times 10^{7}$ & $\cdots$ \\
\hline Dispersion parameter of linear gain $b$ & $1.43 \times 10^{18}$ & $m^{-3} \stackrel{\circ}{A}-2$ \\
\hline Dipole moment $R_{c v}^{2}$ & $6.61 \times 10^{-57}$ & $C^{2} m^{2}$ \\
\hline Value of electron number characterizing nonlinear gain $N_{s}$ & $6.42 \times 10^{7}$ & $\cdots$ \\
\hline Electron life time $\tau_{s}$ & varied & $s$ \\
\hline Refractive index of active region $n_{r}$ & 3.367 & $\cdots$ \\
\hline Effective rate of spontaneous recombination $B_{\text {eff }}$ & $3.50 \times 10^{-16}$ & $m^{3} s^{-1}$ \\
\hline Linewidth enhancement factor $\alpha$ & 2.8 & $\cdots$ \\
\hline Length of the active region $L$ & 300 & $\mu m$ \\
\hline Volume of the active region $V$ & 69 & $\mu m^{3}$ \\
\hline Field confinement factor $\xi$ & 0.2 & $\cdots$ \\
\hline Intraband relaxation time $\tau_{i n}$ & 0.1 & $p s$ \\
\hline Half width of spontaneous emission $\delta \lambda$ & 23 & $n m$ \\
\hline Internal absorption $\kappa$ & 1760 & $m^{-1}$ \\
\hline Front facet power reflectivity $R_{f}$ & 0.3 & $\cdots$ \\
\hline Back facet power reflectivity $R_{b}$ & 0.8 & $\cdots$ \\
\hline
\end{tabular}

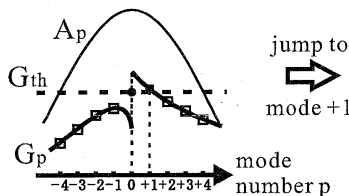

(a)

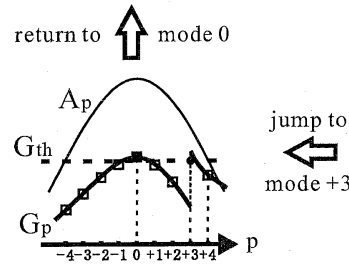

(d)

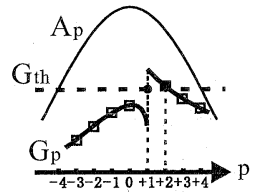

(b)

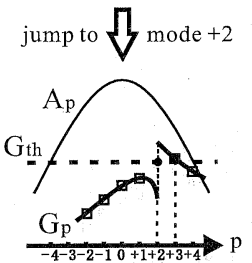

(c)
Fig. 1. Schematic illustration for the rotating effect of the mode hopping among several longitudinal modes. When the asymmetric gain distribution is larger than the linear gain distribution along optical frequencies, the lasing mode hops to the longer wavelength side continuously and hops back to the central mode. The solid circle, the solid square, and the hollow squares mean the operating mode, the mode having higher gain than the threshold, and the nonoscillation mode, respectively.

never exceeds $G_{\mathrm{th}}$. However, when the lasing mode becomes far from the central mode of $p=0$, the lasing gain $G_{p}$ of mode $p=0$ increases again, because of the large value of the linear gain $A_{p}$ and small value of the asymmetric cross-saturation coefficient $H_{p(q)}$. If the gain $G_{p}$ of mode $p=0$ exceeds $G_{\mathrm{th}}$, the lasing mode hops back to the mode $p=0$, as shown in Fig. 1(d) and (a).

The above-mentioned series of hopping is repeated in InGaAsP lasers, where the influence of the asymmetric gain is strong due to a larger value of the asymmetric gain than the differences of the linear gain among the lasing modes. The wave- length range of the hopping and the repeating frequency depend on the material parameters such as the linewidth enhancement factor $\alpha$ and the wavelength dispersion $b$ of the liner gain as well as the wavelength separation $\lambda_{p+1}-\lambda_{p}$ and the injection current $I$.

The lasing operation is the single mode instantaneously. However, the time-averaged lasing spectrum looks like the multimode operation due to continuously repeating mode hopping.

\section{EXPERIMENT}

\section{A. Experimental Setup}

Measurements were done on a buried-heterostructure $(\mathrm{BH})$ type InGaAsP-InP FP laser for 1.3- $\mu$ m emission, in which the transverse mode is well controlled. To confirm experimentally the theoretical prediction, two different setups were prepared.

The first setup was to measure time variations of modal photon number. Each longitudinal mode was separated from other modes by using a monochromator as shown in Fig. 2. Temperature of the laser sample $T$ was fixed at $T=25^{\circ} \mathrm{C}$ with a Peltier element to avoid unnecessary mode-hopping phenomena induced by temperature change. The laser was operated with $I=1.7 I_{\text {th }}$ with $\mathrm{CW}$ current. The output light of each mode was detected by a PIN photodiode and its time variation was examined with a digital oscilloscope. An optical chopper was inserted in front of the monochromator to give identical timing to measure different longitudinal modes with the digital oscilloscope. Measurements were done for modes $p=+1,+2$, and +3 . 


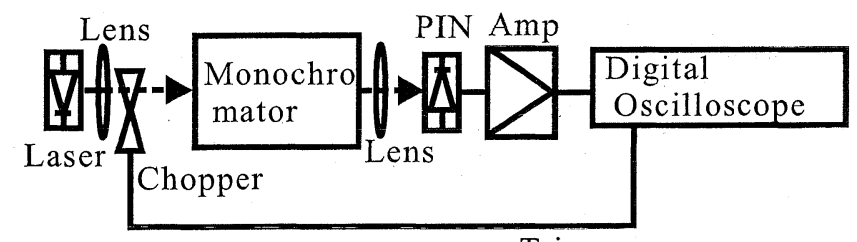

Trigger

Fig. 2. Experimental setup to measure the time variations of the modal photon number.

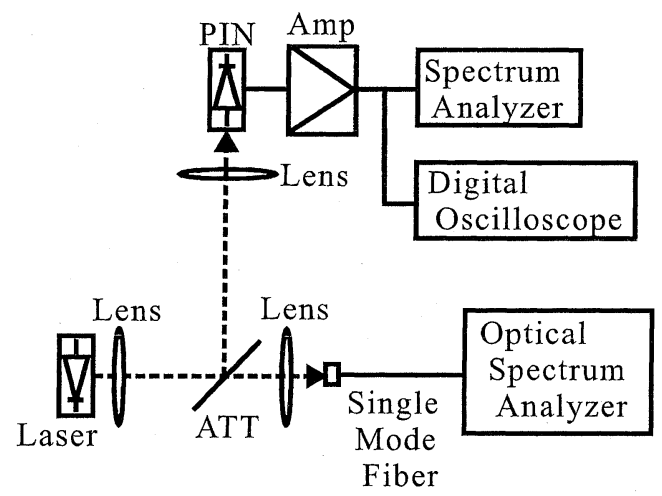

Fig. 3. Experimental setup to measure time variation and frequency spectrum of the total photon number and the time-averaged optical spectrum.

The second setup was to measure time variation and frequency spectrum of total output power over all lasing mode and time-averaged optical spectrum as shown in Fig. 3. The temperature of the laser was also fixed at $T=25^{\circ} \mathrm{C}$. The optical output from the laser was divided by an optical attenuator (ATT). Time variation and frequency spectrum of the total output were examined by using the digital oscilloscope and a spectrum analyzer, respectively. The time-averaged optical spectrum was measured with an optical spectrum analyzer.

\section{B. Time Variations of Modal Photon Number}

The time variations of the modal photon number $S_{p}$ are shown in Fig. 4. In the figure, $\bar{S}$ is the time-averaged total photon number which was examined from measured results given in the next subsection. The figure indicates that the quasi-periodic hopping occurred at almost the same interval but with different initial timing for pulsation. Corresponding theoretical simulations are given in Fig. 5. Rather scattered variations are due to the introduction of the noise sources $F_{p}(t)$ and $F_{N}(t)$ in (1) and (3). Although the measured time variations were smaller than the variations by the theoretical simulations, existence of the quasi-periodic mode hopping was experimentally confirmed.

\section{Time Variation of the Total Photon Number and Longitudinal Mode Spectra}

Experimentally measured time variations and frequency spectrum of the total photon number over all modes are shown in Fig. 6(a) and (b). The driving current level is $I=1.7 I_{\text {th }}$. Time variation of the total photon number indicates almost dc output with small fluctuation, as found in Fig. 6(a). The frequency spectrum is represented in form of the relative intensity noise (RIN) and shows higher values in the range

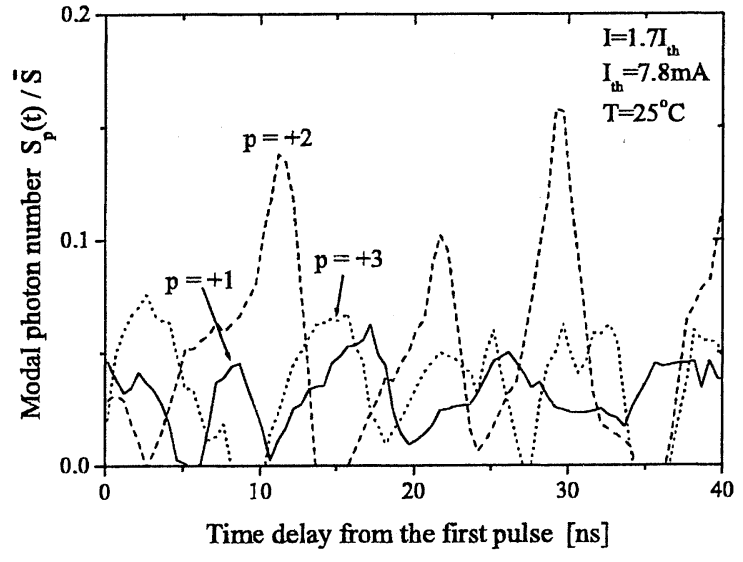

Fig. 4. Experimentally measured time variations of the modal photon numbers.

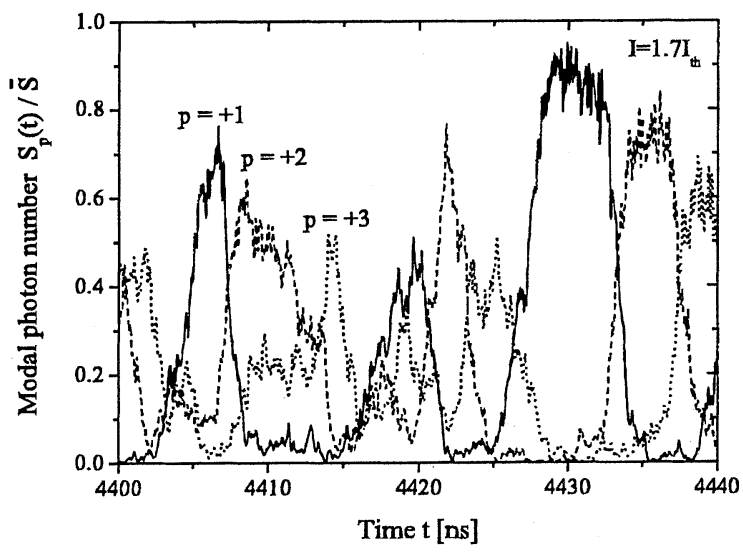

Fig. 5. Simulated results of time variations of the modal photon numbers.

between $50-80 \mathrm{MHz}$. This frequency range may correspond to the repeating frequency of the quasi-periodic mode hopping.

Corresponding theoretical simulations of the time variation and frequency spectrum of the total photon number over all modes are shown in Fig. 7(a) and (b). The time variation shows two types of fluctuations with very rapid variation and rather slow variation. The frequency spectrum in Fig. 7(b) tells us that the fluctuation with rapid variation corresponds to the so-called relaxation frequency at several gigahertz, while the fluctuation with rather slow variation gives another peak in the range between 30-70 MHz. The latter peak corresponds to the repeating frequency of the quasi-periodic mode hopping [10].

In the case of our experimental measurements given in Fig. 6(b), data for the frequency range higher than $170 \mathrm{MHz}$ were not adopted because of inclusions of environmental electromagnetic signals such as TV broadcasters and other experimental equipments into the PIN photodiode. However, we could show evidence of the quasi-periodic mode hopping through frequency spectrum of the total photon number.

Experimentally measured optical spectra are shown in Fig. 8 for cases of (a) to be $I=1.05 I_{\mathrm{th}}$ as an operation near the threshold and (b) to be $I=1.7 I_{\mathrm{th}}$ as another operation with a sufficiently high injection level. In the figures, $\bar{S}_{p}$ is the time-averaged modal photon number and $\lambda_{p}-\lambda_{0}$ indicates wavelength separation between the central mode $p=0$ and a certain mode 

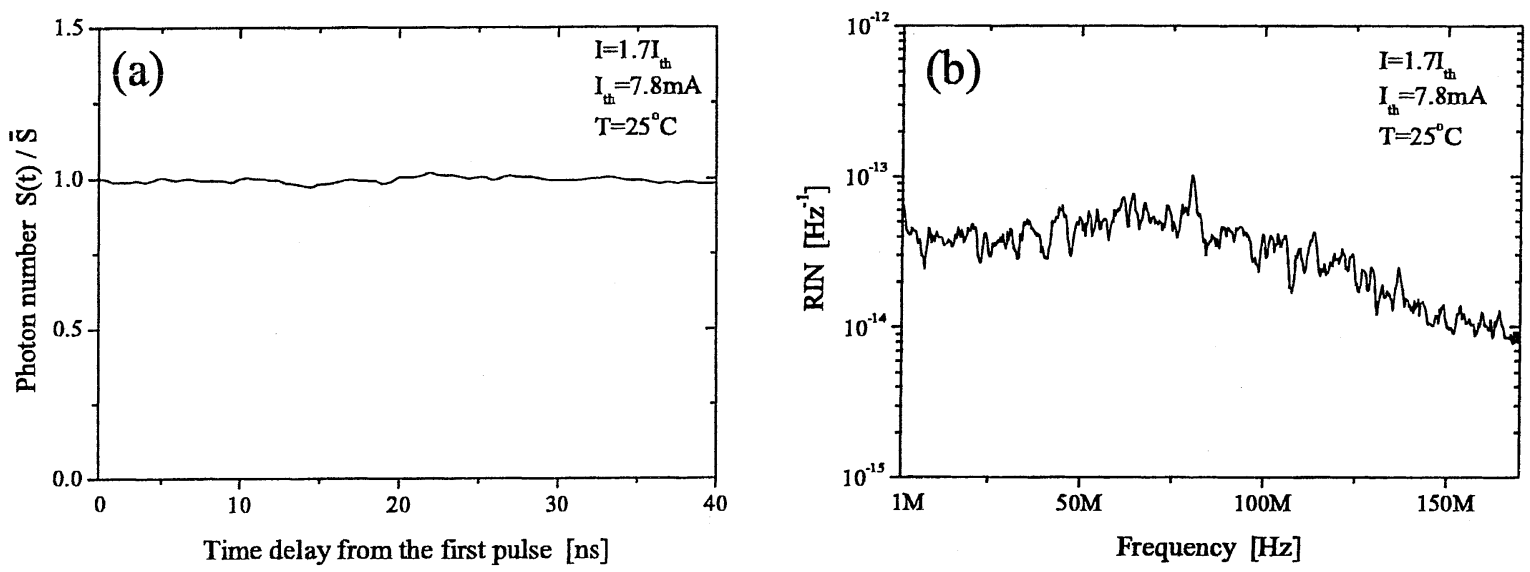

Fig. 6. Experimentally measured results of total photon number. (a) Time variation. (b) Frequency spectrum. The peak of the frequency spectrum in the range from 50 to $80 \mathrm{MHz}$ indicates the quasi-periodic mode hopping.
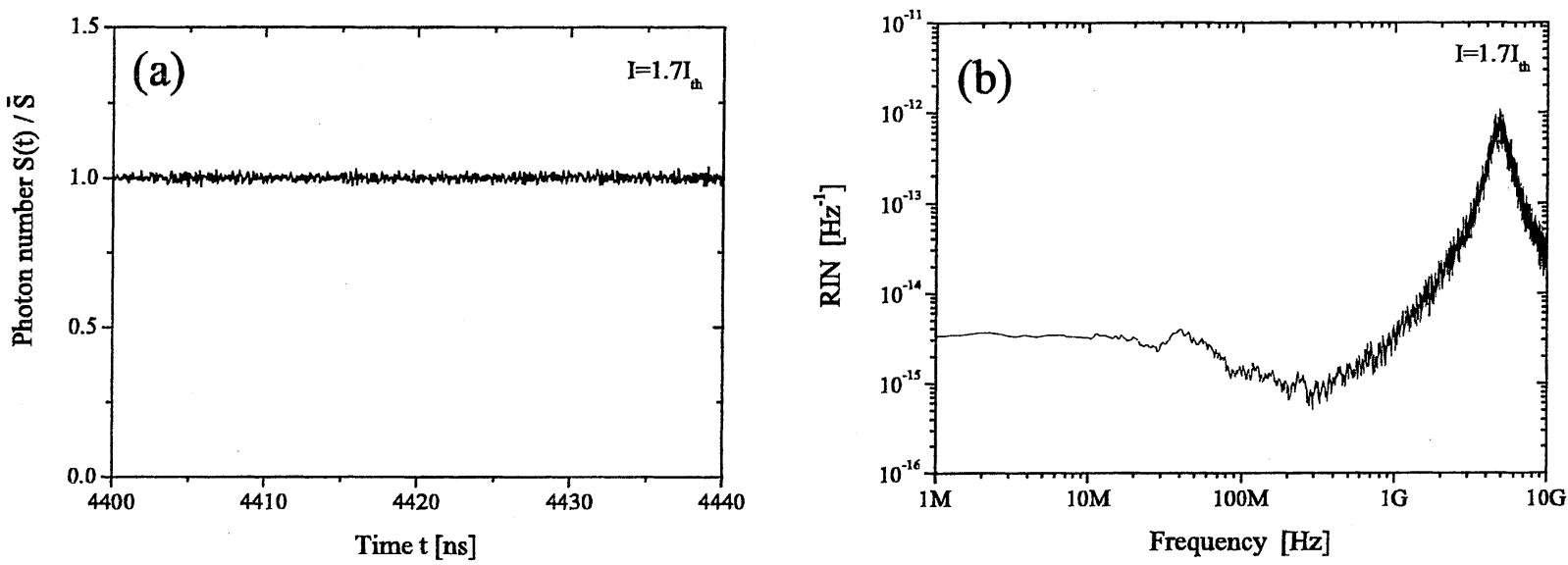

Fig. 7. Simulated results of total photon. (a) Time variation. (b) Frequency spectrum. The peak of the frequency spectrum in the range from 30 to $70 \mathrm{MHz}$ indicates the quasi-periodic mode hopping.
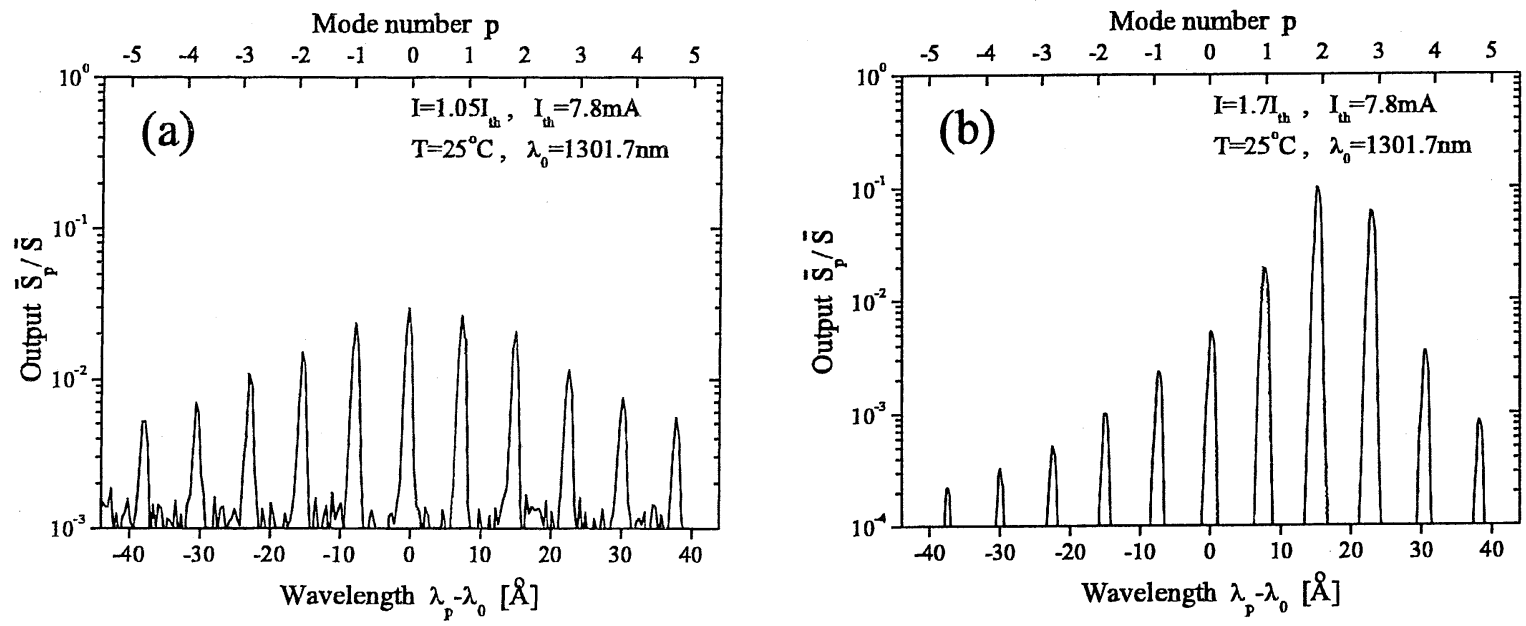

Fig. 8. Experimentally measured optical spectra. (a) At $I=1.05 I_{\mathrm{th}}$. (b) At $I=1.7 I_{\mathrm{th}}$.

$p$. Since the temperature of the laser sample was fixed at $T=$ $25^{\circ} \mathrm{C}$, the wavelength of the central mode must be fixed. Both spectra indicate the multimode operation. However, Fig. 8(a) shows a symmetric profile for variation of the wavelength, while Fig. 8(b) shows an asymmetric profile.
The most dominant mode at $I=1.05 I_{\text {th }}$ is the central mode of $p=0$, but that at $I=1.7 I_{\text {th }}$ shifts to the longer wavelength side up to the mode $p=+2$ in this case. Another remarkable feature at $I=1.7 I_{\mathrm{th}}$ is the asymmetric profile of the modal photon number. The modal photon number on the longer wave- 

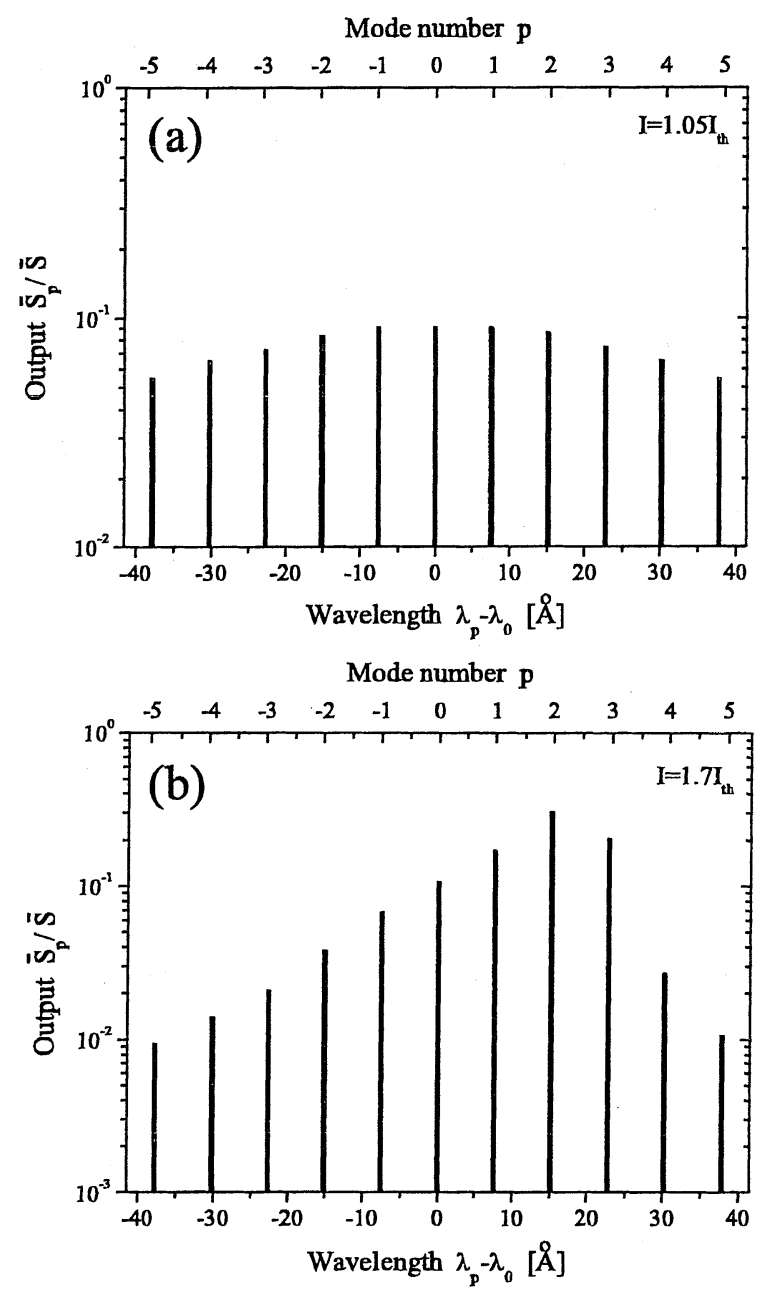

Fig. 9. Simulated results of optical mode spectra. (a) At $I=1.05 I_{\mathrm{th}}$. (b) At $I=1.7 I_{\mathrm{th}}$.

length side reduces more steeply from the dominant mode than those on the shorter wavelength side. These features are also good evidence of the quasiperiodic mode hopping.

Theoretically simulated results corresponding to Fig. 8(a) and (b) are given in Fig. 9(a) and (b). When the injection current is very near to the threshold current level such as $I=1.05 I_{\text {th }}$ inclusion of the spontaneous emission is still strong, resulting in stable multimode operation [2], [6]. However, when the injection current increases more, the quasi-periodic mode hopping starts, resulting in multimode-like profiles in the time-averaged spectrum with an asymmetric profile for wavelength distribution.

\section{CONCLUSION}

In this paper, we demonstrated experimental evidences of the timely repeated mode-hopping phenomena in an InGaAsP-InP FP laser for $1.3-\mu \mathrm{m}$ emission by tracing time-dependent variations of lasing modes and compared the results with theoretical simulations.

Our obtained results are as follows.

1) When the laser was operated with the high injection current level, the mode-hopping occurred repeatedly among several longitudinal modes even when the total output was almost constant.

2) The aforementioned hopping resulted in multimode-like characteristics in the time-averaged optical spectrum but its profile was asymmetric for wavelength distribution.

3) The frequency spectrum of the total photon number over all modes had a peak in the range from 50 to $80 \mathrm{MHz}$ which corresponds to the rotation frequency of the hopping modes.

4) The features of the experimental results were well supported with the theoretical simulations.

\section{ACKNOWLEDGMENT}

The authors acknowledge Dr. S. Ogita and Dr. T. Higashi of Fujitsu Quantum Devices Ltd. for supplying the laser samples and discussions.

\section{REFERENCES}

[1] M. Yamada, K. Hayano, H. Ishiguro, and Y. Suematsu, "An approximate analysis of gain suppression in injection lasers for band-to-band and band-to-impurity-level transitions," Jpn. J. Appl. Phys., vol. 18, pp. 1531-1541, 1979.

[2] M. Yamada and H. Nagato, "Analysis of longitudinal mode behavior around the threshold level in undoped injection lasers," Trans. Inst. Electron. Commun. Eng. Japan, vol. E64, pp. 770-777, 1981.

[3] M. Yamada, "Transverse and longitudinal mode control in semiconductor injection lasers," IEEE J. Quantum Electron., vol. QE-19, pp. 1365-1380, 1983.

[4] M. Nakamura, K. Aiki, N. Chinone, R. Ito, and J. Umeda, "Longitudinal-mode behaviors of mode-stabilized $\mathrm{Al}_{x} \mathrm{Ga}_{1-x} \mathrm{As}$ injection lasers," J. Appl. Phys., vol. 49, pp. 4644-4648, 1978.

[5] M. Yamada and Y. Suematsu, "A condition of single longitudinal mode operation in injection lasers with index-guiding structure," IEEE J. Quantum Electron., vol. QE-15, pp. 743-749, 1979.

[6] - "Analysis of gain suppression in undoped injection lasers," $J$. Appl. Phys., vol. 52, pp. 2653-2664, 1981.

[7] I. Mito, M. Kitamura, K. Kaede, Y. Odagiri, M. Seki, M. Sugimoto, and K. Kobayashi, "InGaAsP planer heterostructure laser diode (PBH-LD) with very low threshold current," Electron. Lett., vol. 18, pp. 2-3, 1982.

[8] J. Manning, R. Olshansky, D. M. Fye, and W. Powazinik, "Strong influence of nonlinear gain on spectral and dynamic characteristics of InGaAsP lasers," Electron. Lett., vol. 12, pp. 496-497, 1985.

[9] S. Ogita, A. J. Lowery, and R. S. Tucker, "Influence of asymmetric nonlinear gain on the transient of longitudinal modes in long wavelength Fabry-Perot laser diodes," IEEE J. Quantum Electron., vol. 33, pp. 198-210, 1997.

[10] M. Ahmed and M. Yamada, "Influence of instantaneous mode competition on the dynamics of semiconductor lasers," IEEE J. Quantum Electron., vol. 38, pp. 682-693, 2002.

[11] H. Ishikawa, M. Yano, and M. Takusagawa, "Mechanism of asymmetric longitudinal mode competition in InGaAsP/InP lasers," Appl. Phys. Lett., vol. 40, pp. 553-555, 1982.

[12] N. Ogasawara and R. Ito, "Longitudinal mode competition and asymmetric gain saturation in semiconductor injection lasers: I. Experiment," Jpn. J. Appl. Phys., vol. 27, pp. 607-614, 1988.

[13] — , "Longitudinal mode competition and asymmetric gain saturation in semiconductor injection lasers: II. Theory," Jpn. J. Appl. Phys., vol. 27, pp. 615-626, 1988.

[14] M. Yamada, "Theoretical analysis of nonlinear optical phenomena taking into account the beating vibration of the electron density in semiconductor lasers," J. Appl. Phys., vol. 66, pp. 81-89, 1989.

[15] - "Theory of mode competition noise in semiconductor lasers," IEEE J. Quantum Electron., vol. QE-22, pp. 1052-1059, 1986.

[16] C. H. Henry, "Phase noise in injection lasers," J. Lightwave Technol., vol. LT-4, pp. 298-311, 1986.

[17] M. Ahmed, M. Yamada, and M. Saito, "Numerical modeling of intensity and phase noises in semiconductor lasers," IEEE J. Quantum Electron., vol. 37, pp. 1600-1610, 2001. 


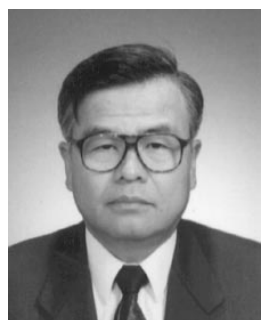

Minoru Yamada (M'82) was born in Yamanashi, Japan, on January 26, 1949. He received the B.S. degree in electrical engineering from Kanazawa University, Kanazawa, Japan, in 1971 and the M.S. and $\mathrm{Ph} . \mathrm{D}$. degrees in electronics engineering from the Tokyo Institute of Technology, Tokyo, Japan, in 1973 and 1976, respectively

He joined Kanazawa University in 1976, where he is presently a Professor. From 1982 to 1983, he was a Visiting Scientist at Bell Laboratories, Holmdel, NJ. He is currently doing research work on semiconductor injection lasers, semiconductor optical switches, and unidirectional optical amplifiers.

Dr. Yamada received the Yonezawa Memorial Prize in 1975, the Paper Award in 1976, and the Achievement Award in 1978 from the Institute of Electronics and Communication Engineers of Japan.

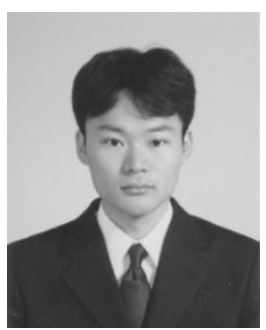

Wataru Ishimori was born in Ishikawa, Japan, on October 21, 1978. He received the B.S. degree in electrical and computer engineering from Kanazawa University, Kanazawa, Japan, in 2001. He is presently working toward the M.S. degree at the same university.
Hironobu Sakaguchi was born in Kyoto, Japan, on September 23, 1979. He received the B.S. degree in electrical and computer engineering from Kanazawa University, Kanazawa, Japan, in 2002. He is presently working toward the M.S. degree at the same university.

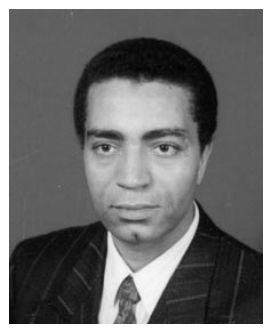

Moustafa Ahmed (S'99-M'99) was born in Minia, Egypt, in 1966. He received the B.Sc. and M.Sc. degrees in physics from Minia University, Minia, Egypt, in 1988 and 1993, respectively, and the Ph.D.Eng. degree from Kanazawa University, Kanazawa, Japan, in 1999. His dissertation research mainly involved design methods for optical filters and an infinite-order approach to gain calculation in semiconductor lasers

$\mathrm{He}$ is currently a Lecturer in the Physics Department, Minia University. He was a Visiting Fellow at the Department of Electrical and Electoronic Engineering, Kanazawa University, supported by the postdoctoral program of Japan Society for Promotion of Science (JSPS). His research interests are in the areas of optoelectronics and statics and dynamics of semiconductor lasers. 